\title{
Pathogenesis of necrotising enteritis with special reference to intestinal hypersensitivity reactions
}

\author{
S N ARSECULERATNE, R G PANABOKKE, AND C NAVARATNAM
}

\begin{abstract}
From the Departments of Microbiology and Pathology, Faculty of Medicine, University of Peradeniya, Sri Lanka
\end{abstract}

SUMMARY The aetiological aspects of 83 sporadic cases of necrotising enteritis (NE) have been studied. Of 56 cases in which histology of the intestine was possible, eight showed appearances (oedema and local eosinophilia) suggestive of a type I hypersensitivity reaction, while in 37 the appearances were suggestive of a type III reaction. We suggest that these reactions, which were more common in children and young adults, were initiating factors in the intestinal necrosis. The type III reactions (submucosal arteritis, fibrinoid necrosis of arteriolar walls, intramural and perivascular infiltration with polymorphonuclear, mononuclear, and eosinophil cells, and submucous oedema) were in seven cases accompanied by extraintestinal lesions (hypercellularity of glomeruli, amorphous material in the Bowman's capsular space, tubular casts, mononuclear cell infiltration into the hepatic portal tracts, congestion and oedema of the lung) which were compatible with systemic immune complex disease. The mesenteric lymph nodes in 12 out of 15 cases with intestinal arteritis showed appearances indicative of a humoral immune response. We suggest that NE is a two-stage process. In stage 1, a necrotic focus is established in the intestinal mucosa-submucosa by 'initiating' factors of vascular (functional or organic) or microbial (exotoxic, endotoxic, or Shwartzman) origin. Functional circulatory insufficiency in the intestine is of particular relevance to necrotising enteritis in neonates and in adults with traumatic shock or cardiac insufficiency. The jejunal and-to a lesser extent-the ileal microcirculation appear to be particularly vulnerable to microcirculatory insufficiency. Ninety-seven per cent of our cases were of NE of the small intestine of which $76 \%$ involved the jejunum alone or as a part of a jejunoileitis. These 'initiating' factors act either singly or synergistically with 'promoting' factors (changes in the volume, composition, or $\mathrm{pH}$ of the diet, intestinal stasis, or bacterial factors) in the establishment of necrotic foci in the intestine. Stage 2 results from the colonisation of the necrotic foci by intestinal clostridia, the toxigenic capacity of which will determine the progress of the intestinal lesion. Clinically established NE is essentially gas gangrene of the intestinal wall. Our bacteriological findings (microscopic, cultural, and serological) support a pathogenetic role of $\mathrm{Cl}$. welchii in the established stages of necrotising enteritis. Strains of $\mathrm{Cl}$. welchii from NE cases had significantly higher histidine decarboxylase activity than strains from control sources; it is possible that the resultant histamine production could act as a promoting factor in stage 1 . Neutralising antibody against the Wanowrie virus, an Asian arbovirus which produces haemorrhagic enteritis in mice, was absent in the paired sera from 10 cases examined.

In an extensive literature, necrotising enteritis (NE) has been described under various synonyms such as acute segmental necrotising enteritis, necrotising enterocolitis, enteritis necroticans, and acute segmental ischaemic enteritis. It refers to a clinicopathological entity of an acute abdominal disorder which is characterised by patchy or segmental necrosis of varying extent of the wall of the jejunum,

Received for publication 10 October 1979 ileum, or colon and which is sometimes accompanied by intestinal gangrene, toxaemia, and death in an appreciable proportion of cases. Watery diarrhoea and suppuration are not features of this entity.

Sporadic and epidemic cases have been described from diverse countries. There has also been a wide spectrum of associated conditions and clinical features in an equally wide range of patients including stressed or premature neonates, children, and adults with apparently no previous abnormality 
and patients with various preceding and possibly determinant conditions such as intestinal infections, shock or surgical operations, and states of circulatory insufficiency. These latter states appear to have had pathophysiological factors which precipitated the intestinal lesions.

We have seen similar cases of necrotising enteritis, although epidemics have not been encountered. The sporadic incidence, however, seems to be increasing in some parts of Sri Lanka. Except for brief clinical descriptions ${ }^{1-3}$ no documentation of the pathology or aetiology of Sri Lankan cases has hitherto been made.

This report describes the aetiological aspects of 83 cases which were admitted to the main state hospital of this district, and which were diagnosed at laparotomy or necropsy, either macroscopically or microscopically with resected specimens of the affected intestine. Our cases were classified on criteria which are similar to those of Murrell et al.4: type Ifulminant, acute cases with a history of two days or less ending in death within two days of admission; type II-acute surgical cases with a history of two to 14 days, which needed laparotomy with or without resection; type III-subacute surgical cases, with milder symptoms of two weeks or more in duration and which were treated surgically after conservative treatment had failed. As only those cases which were diagnosed at laparotomy or with resected specimens were studied, type IV cases which were treated conservatively have been excluded from this series. The cases described here are from a larger series studied by Kumarakulasinghe and Ratnatunga ${ }^{3}$ from the viewpoints of clinical presentation and management.

We suggest from our findings that local hypersensitivity reactions of type I and type III are also 'initiating' factors. As far as we are aware, no previous description of an immunological origin of vascular determinants in necrotising enteritis has hitherto been made. Non-immunological release of histamine by intestinal helminths and bacteria may also contribute to the microcirculatory disturbances.

\section{Methods}

PATIENTS AND CONTROL SUBJECTS

The patients with necrotising enteritis were either admitted directly or were referred for specialised treatment from neighbouring hospitals. Eighty-three cases (57 males and 26 females) were studied including 47 children under 15 years of age. In only some of these patients was it possible to do all the investigations described.

Patients with non-intestinal diseases-for example, uncomplicated fractures-in the same wards and blood donors served as controls for serology and intestinal strains of Clostridium welchii.

\section{HISTOPATHOLOGY}

Pathological portions of intestine, resected at laparotomy or necropsy as early as possible after death, were fixed together with macroscopically normal pieces of adjacent segments of the intestine in $10 \%$ formol saline. Mesenteric lymph nodes were similarly treated. Paraffin sections were stained with haematoxylin and eosin ( $\mathrm{H}$ and $\mathrm{E})$ and Gram's stain. Histological evaluation of the immune response in the lymph nodes was made according to the system proposed by Cottier and his colleagues. ${ }^{5}$

\section{BACTERIOLOGICAL STUDIES}

Smears of intestinal contents aspirated from the pathological segments of the intestine and homogenates of mesenteric lymph nodes in isotonic saline were stained by Preston and Morrell's modification of the Gram stain.

In view of the evidence in the literature for the role of $\mathrm{Cl}$. welchii in necrotising enteritis, selective isolation procedures were adopted for the recovery of this organism from the intestinal contents of NE patients and the faeces of controls and from the mesenteric node homogenates in sterile isotonic saline. These were inoculated into Robertson's cooked meat broth (Oxoid) a parallel bottle of which was heated in a boiling water bath for 10 minutes. Sheep blood agar plates inoculated with the same material were incubated aerobically and anaerobically.

Standard bacteriological procedures ${ }^{6}$ were used for the isolation, biochemical characterisation, and identification of isolates.

$\mathrm{Cl}$. welchii was typed by the toxin-antitoxin neutralisation test in guinea-pigs' skin using standard $\mathrm{Cl}$. welchii antitoxins (Wellcome Diagnostic Reagents) with filtrates from cultures in liver extractcooked meat broth.

Histidine decarboxylase activity was measured as follows. The centrifuged deposit from one day old cultures of $\mathrm{Cl}$. welchii in Oxoid cooked meat broth was washed thrice with distilled water at $4^{\circ} \mathrm{C}$ and finally resuspended in distilled water to a density of $10 \mathrm{mg} / \mathrm{ml}$ dry weight. The suspensions were stored at $4^{\circ} \mathrm{C}$ during the weight determinations. Enzyme activity was measured in Warburg manometers, in duplicate, with l-histidine in $0.1 \mathrm{M}$ acetate buffer (pH 4.5) at $28^{\circ} \mathrm{C} .^{7}$

Serological tests were done on paired sera; the first specimen was collected soon after admission and the second just before the patient was discharged. The sera were stored at $4^{\circ} \mathrm{C}$ for examination or were 
lyophilised or stored at $-20^{\circ} \mathrm{C}$ when tests were delayed.

Sera for viral neutralisation tests were examined by Dr K M Pavri (Virus Research Centre, Poone, India) for neutralising antibodies against the Wanowrie virus. This is an arbovirus prevalent in Asia and which produces haemorrhagic enteritis in baby mice.

Titration of $C$. welchii antitoxins was restricted to the anti $\alpha$ toxin, as the predominant type isolated was type A. The haemolytic method with washed rabbit red cells was used with a freeze dried sample of ammonium sulphate precipitated toxin from the culture supernatant of a highly toxigenic type A strain, grown in liver extract-cooked meat broth. A standard anti $\alpha$ toxin of known unitage was used as reference.

Student's $t$ test was used in the assessment of significance.

\section{Results}

SEASONAL INCIDENCE

Our cases of necrotising enteritis occurred during the dry intermonsoonal months of June to October with a peak incidence in August-September. This pattern contrasts with that of gastroenteritis in children in this district, due to Salmonella, Shigella, and Escherichia coli (Arseculeratne, unpublished observations); the latter cases were most prevalent during

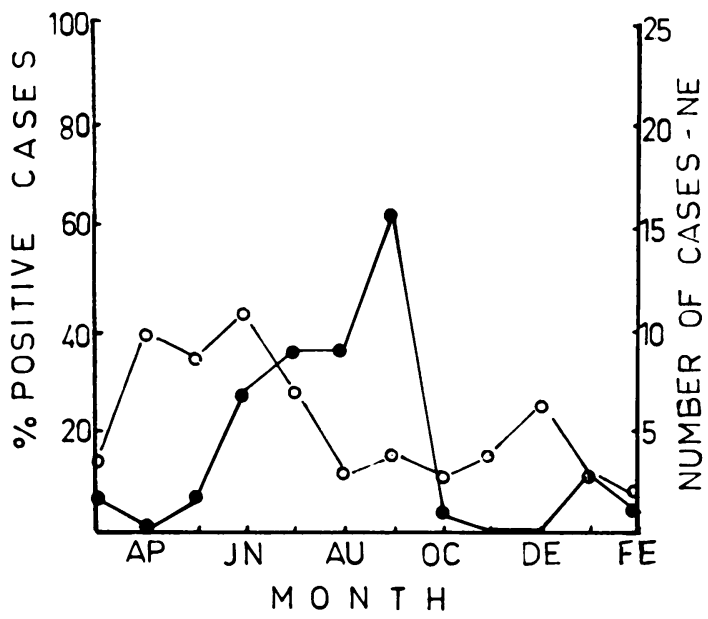

Fig. 1 The mean, monthly incidence of gastroenteritis (-O-) due to Salmonella, Shigella and E. coli as a percentage of the total number of cases examined compared with the total incidence of necrotising enteritis (-०) in a three-year period. and just after the wet months of March-July and October-January (Fig. 1). There were no epidemics.

\section{AGE AND SEX DISTRIBUTION}

The ages ranged from 11 days to 65 years with a sex distribution of 56 males and 26 females. As our series excluded those patients of clinical type IV where confirmation of the diagnosis was not possible, these figures do not represent the true age and sex distribution.

\section{DIET}

Our patients included vegetarians as well as those who consumed meat and fish. There was no particular item of food which was associated with the onset of the illness. We particularly inquired about this consumption of sweet potato because of the suggestion $^{8}$ that trypsin inhibitors from this yam could potentiate the action of clostridial toxins by preventing their degradation. No association between sweet potato and necrotising enteritis was evident.

\section{PRECEDING ILLNESSES}

Three patients had a preceding illness or abnormality which was probably the precipitating factor in the enteritis; these were two adult males, one of whom had cardiac insufficiency and the other had hypotension and shock after a fall from a tree. The third case was a premature baby, 11 days old. None of the patients showed clinical signs of malnutrition.

\section{CLINICAL TYPES}

The type distribution was as follows: type $1,34 \%$; type II, $60 \%$; type III, $6 \%$.

The distribution according to the site of intestinal lesions and age category in 68 cases in which records were available is shown in Table 1 .

\section{BACTERIOLOGICAL FINDINGS}

In 21 of the 27 cases of jejunitis and upper ileitis that were examined Gram stained smears of contents aspirated from the pathological intestine showed large Gram positive bacilli which morphologically resembled clostridia (Fig. 2). In many cases of upper jejunitis these bacteria were the only forms seen, and pure cultures of $C$. welchii were recovered on culture of the contents.

Table 1 Distribution according to site and age category

\begin{tabular}{lllcl}
\hline & Jejunum & $\begin{array}{l}\text { Jejunum and } \\
\text { upper ileum }\end{array}$ & Ileum & $\begin{array}{l}\text { Lower ileum and } \\
\text { ascending colon }\end{array}$ \\
\hline Adults & 14 & 6 & 10 & 1 \\
Children & 18 & 12 & 6 & 1 \\
Total & 32 & 18 & 16 & 2 \\
\hline
\end{tabular}


Histologically in two cases, in areas of necrotic mucosa and submucosa, masses of similar bacilli were adherent to the necrotic tissues (Fig. 3); these bacilli were also present in the adjacent submucosa (Fig. 4). In cases of lower ileitis, the intraluminal contents showed a mixed bacterial flora of Gram negative bacilli and the larger Gram positive forms. The exclusive presence of the latter within the submucosa suggests an invasion by these bacilli of the submucosal tissue. In segments of the adjacent relatively normal intestine, these bacilli were absent on the mucosal lining and in the deeper tissues.

No bacilli were seen in the mesenteric lymph
Table 2 Type distribution of $\mathrm{Cl}$. welchii cases

\begin{tabular}{|c|c|c|c|c|c|c|}
\hline & \multicolumn{6}{|c|}{ Type } \\
\hline & $A$ & $B$ & $C$ & $\begin{array}{l}\text { A and } \\
\text { nontypable }\end{array}$ & $\stackrel{A}{C}$ and & Nontypable \\
\hline Number of cases & 4 & 2 & 0 & 4 & 1 & 1 \\
\hline
\end{tabular}

nodes, even in cases with extensive gangrene of the intestine.

Culturally, 12 out of 27 cases yielded $\mathrm{Cl}$. welchii from the intestinal contents. The type distribution of these strains is shown in Table 2.

None of the isolates had heat resistant spores. The

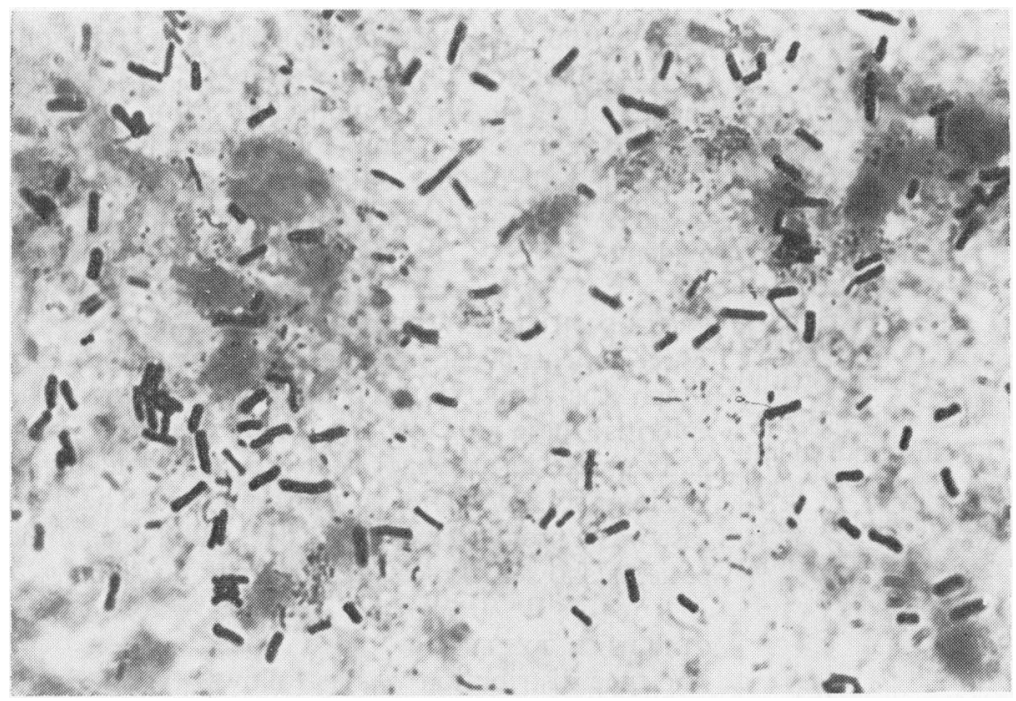

Fig. 2 Gram stained smear of contents of necrotic jejunum. Note almost exclusive presence of bacilli resembling clostridia. $\times 625$. (All figures show original magnification.)

Fig. 3 Bacilli (arrows) morphologically resembling clostridia adherent to necrotic mucosa in necrotising jejunitis. $H$ and $E, \times 1040$.

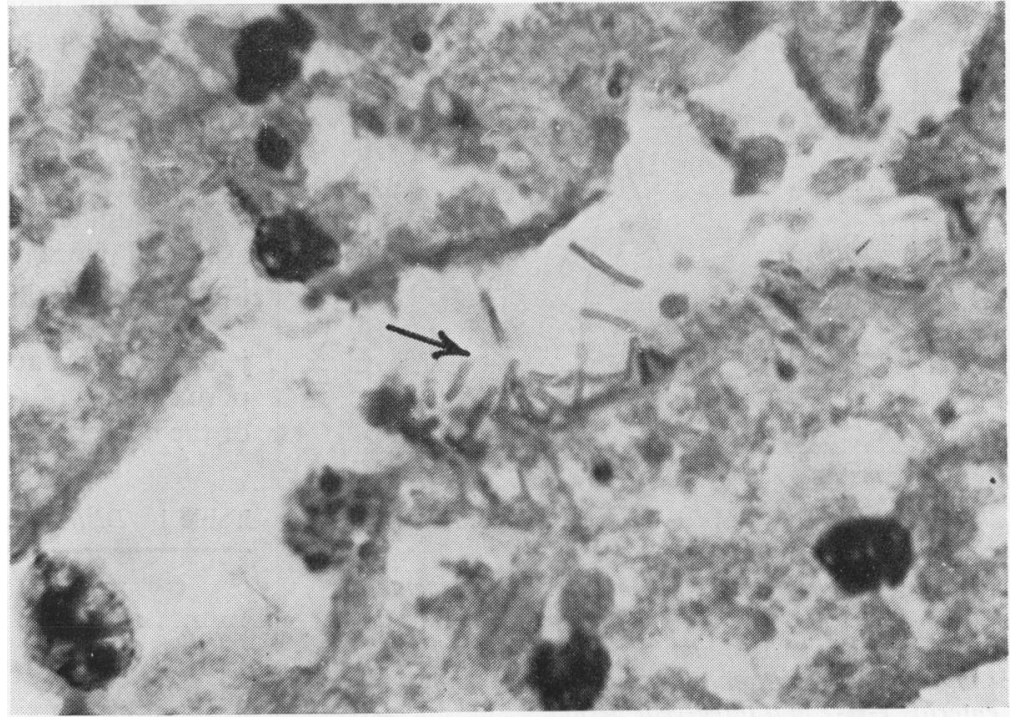


Table 3 Comparison of strains of $\mathrm{Cl}$. welchii from patients with necrotising enteritis and from control subjects in histidine decarboxylase activity (30 minutes incubation $\left.28^{\circ} \mathrm{C}, \mathrm{pH} 4.5(\mathrm{P}<0.05)\right)$

\begin{tabular}{lll}
\hline & \multicolumn{2}{l}{ Strains } \\
\cline { 2 - 3 } & Necrotising enteritis & Control \\
\hline Number & 20 & 15 \\
$\mu \mathrm{l} \mathrm{CO}$ liberated & $5 \cdot 45 \pm 5.5$ & $2 \cdot 48 \pm 1.88$ \\
\hline
\end{tabular}

Table 4 Titres of anti a toxin $(\mathrm{Cl}$. welchii) in units $/ \mathrm{ml}$ serum in necrotising enteritis patients and control subjects $(\mathrm{P}<0.001)$

\begin{tabular}{lllr}
\hline & Number & \multicolumn{2}{l}{ Titres } \\
\cline { 3 - 4 } & & Range & Mean \\
\cline { 3 - 4 } & & $0 \cdot 0-3 \cdot 3$ & $1 \cdot 1 \pm 0 \cdot 7$ \\
Controls & 40 & $2 \cdot 2-48 \cdot 8$ & $11 \cdot 8 \pm 9 \cdot 5$ \\
Necrotising enteritis & 26 & & \\
Patients & & & \\
\hline
\end{tabular}

mesenteric nodes in all 18 cases examined were sterile.

\section{Histidine decarboxylase activity}

Liberation of $\mathrm{CO}_{2}$ from histidine by strains of $\mathrm{Cl}$. welchii from control sources and patients with necrotising enteritis is shown in Table $3 . \mathrm{CO}_{2}$ liberation by NE strains was significantly $(\mathrm{P}<0.05)$ greater than by control strains at the end of 30 minutes incubation.

\section{SEROLOGICAL FINDINGS}

\section{Anti $\alpha$ toxin titres}

Table 4 summarises the titres in the sera of patients with necrotising enteritis and in controls. A significant rise of titres in NE patients $(\mathrm{P}<0.001)$ was noted.

\section{Arboviral neutralising antibody}

None of the paired sera from 10 cases of necrotising enteritis had neutralising antibody against the Wanowrie virus.

\section{PATHOLOGICAL FINDINGS \\ Macroscopic}

In mild and moderate cases, the intestinal lesions were confined to circumferentially distributed areas in the jejunum or ileum in lengths of gut varying from 1 to $5 \mathrm{~cm}$; these areas were congested or plum coloured, haemorrhagic, and oedematous. Often, several centimetres of the intestine were affected.

The affected areas were separated by intervening portions of less affected or normal intestine, thus giving the appearance of 'skipped lesions'. The mesentery appeared normal and the mesenteric vessels were patent and pulsatile. The mesenteric nodes were soft and enlarged.

In more advanced cases with extensive haemorrhagic necrosis and oedema in the wall with denudation of the mucosa, the mesentery appeared oedematous and haemorrhagic with blood-tinged fluid in the peritoneal cavity.

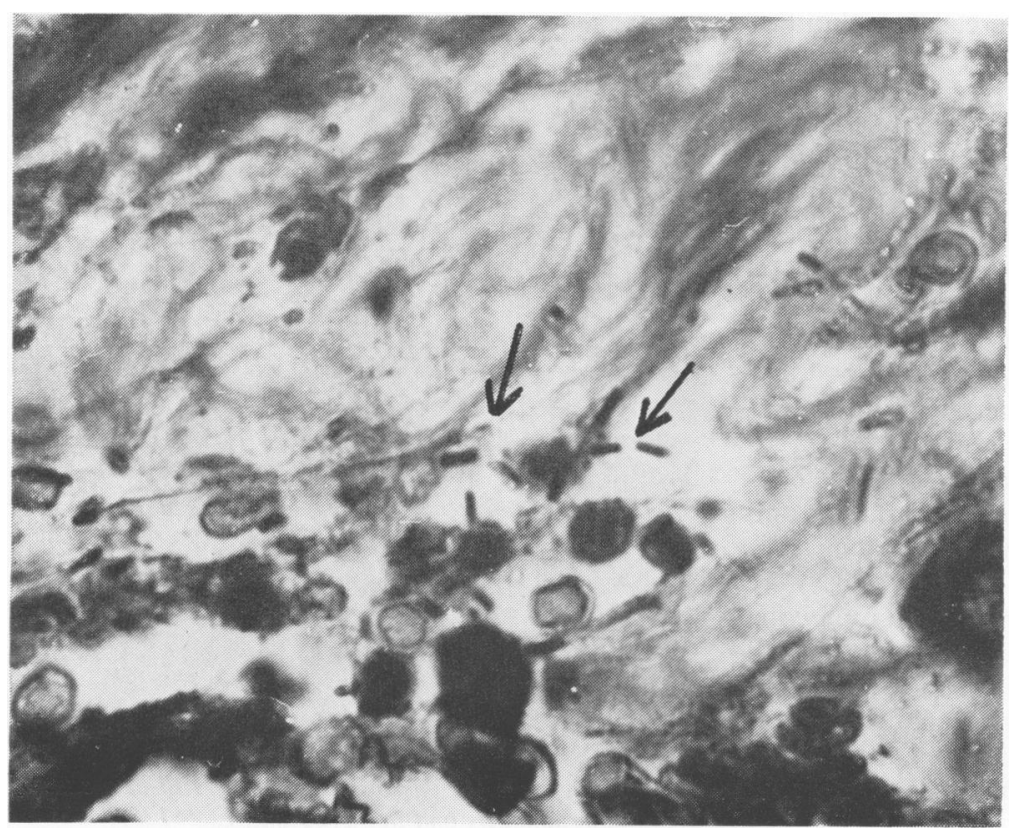

Fig. 4 Bacilli morphologically resembling clostridia in necrotic submucosa. Necrotising jejunitis. $H$ and $E, \times 1040$. 


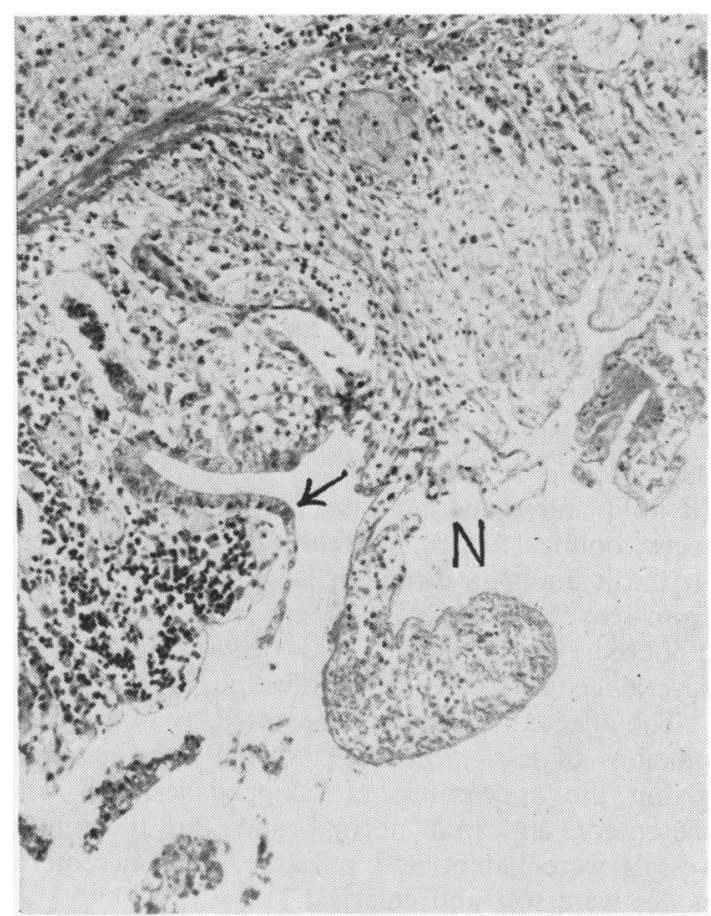

Fig. 5 Focal necrosis ( $N$ ) of mucosa adjacent to relatively normal mucosa (arrow). Necrotising ileitis. $H$ and $E, \times 160$.

\section{Microscopic findings}

Histological material was available in 56 cases. The following features were seen in cases without a preceding or associated illness.

Necrosis This appeared to be focal in origin (Fig. 5) starting at the tips of the villi with oedema and vascular congestion of the adjacent submucosa. Severe congestion was noted in some villi, especially at the villous tips (Fig. 6). Extension of the necrosis appeared to be centrifugal to the submucosa, muscularis mucosae and the muscle layers.

Cell infiltration Where cellular reactions and vascular abnormalities were concerned, three patterns were noted.

1 The cell infiltrate was most marked in the submucosa and consisted of polymorphonuclear and mononuclear cells and eosinophils in varying proportions. Large accumulations of these cells were mainly in the perivascular regions accompanying the vascular lesions described below. Infiltration of the submucosa with eosinophils in the vicinity of the necrosis was seen in 17 cases; perivascular collections of eosinophils were prominent around the smaller vessels, which showed arteritis and fibrinoid necrosis (Fig. 7).
The vascular lesions were confined to the smaller submucosal arteries, while the veins appeared to be relatively normal (Fig. 8). These consisted of oedema and cell infiltration into the wall and perivascularly and a loss of fibrillar structure of the wall, which appeared homogeneous or granular and eosinophilic, giving the appearance of fibrinoid necrosis. Vasculitis with intramural cell infiltration was seen in seven cases, while fibrinoid necrosis was seen in 14 cases. Twelve cases showed both cell infiltration murally and perivascularly with fibrinoid necrosis. Occasionally the cell infiltration and oedema of the wall of the artery were segmental.

The mural and perivascular cell infiltrate was predominantly polymorphonuclear in seven cases and was both polymorphonuclear and mononuclear in 15 cases and predominantly mononuclear (Fig. 9) in eight. A predominantly eosinophil cell infiltrate with fibrinoid necrosis was seen in four cases. Fibrinoid necrosis without marked cell infiltration of the wall and the immediate perivascular region, was noted in two cases.

Some of the small veins in these pathological areas showed hyaline thrombi with partial or total occlusion of the lumen (Fig. 10).

These vascular abnormalities (mural oedema with

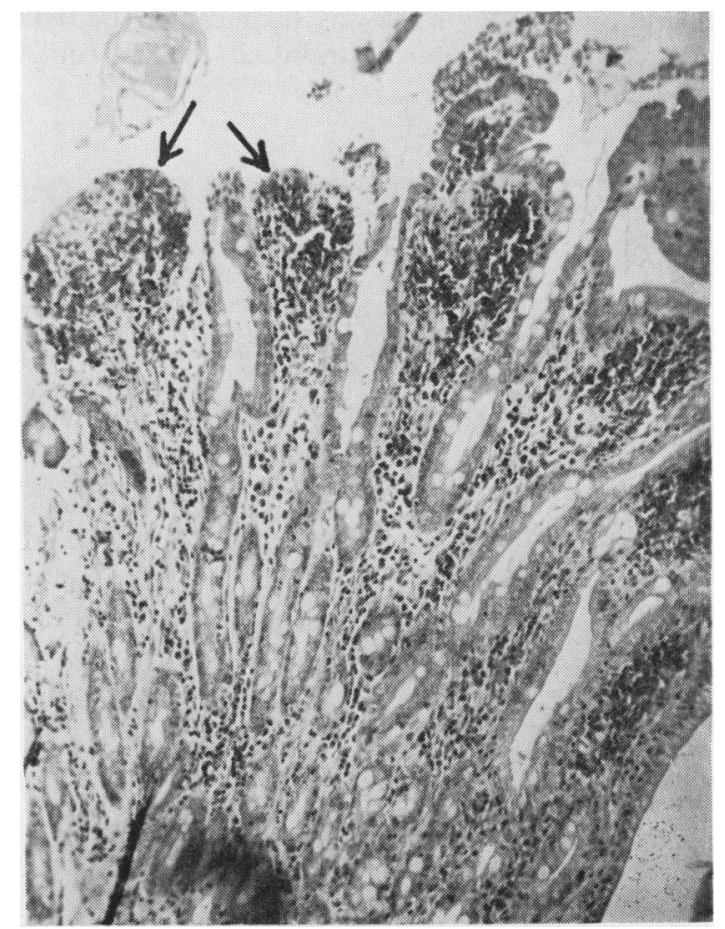

Fig. 6 Hyperaemia (arrows) at tips of villi in a case of necrotising jejunitis and ileitis. $H$ and $E, \times 125$. 


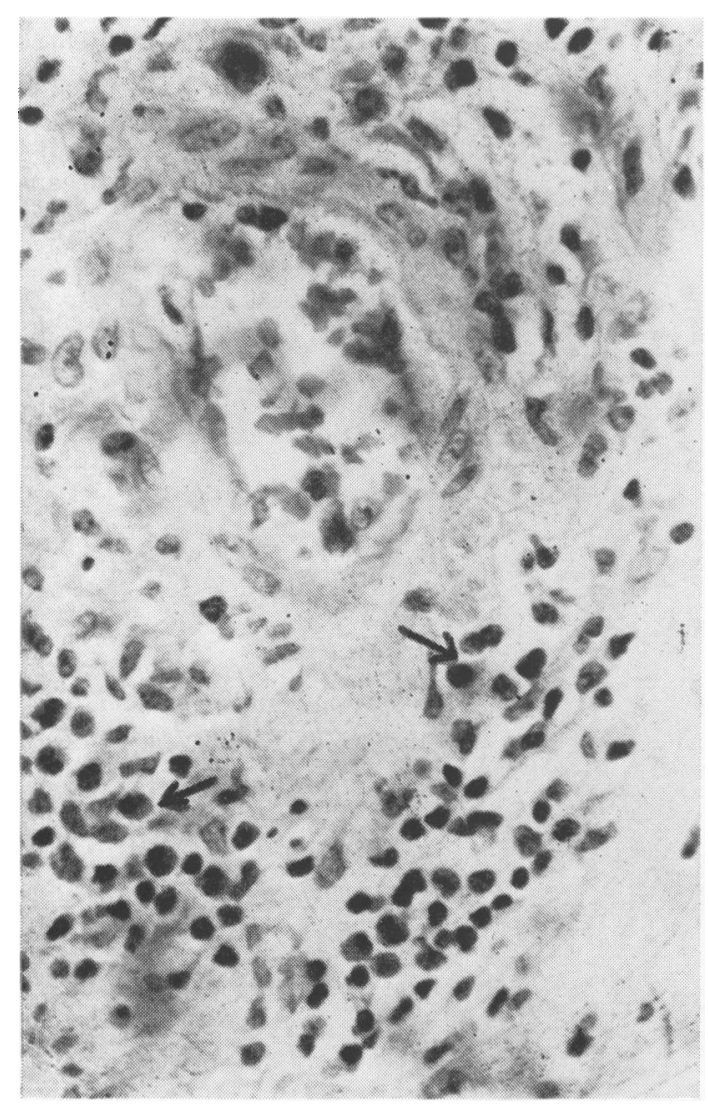

Fig. 7 Perivascular infiltration of eosinophils (arrows) adjacent to submucosal artery showing fibrinoid necrosis. Necrotising jejunitis in a 22-year-old male.

$H$ and $E, \times 625$. cell infiltration, fibrinoid necrosis, and perivascular cell infiltration) were confined to the small arteries, while the veins were relatively unaffected (Fig. 8) and were suggestive of a type III hypersensitivity reaction. They were seen especially in older children and adults, except in the old patients with associated cardiovascular abnormalities.

2 A second pattern of histopathology in eight patients consisted of necrosis of the mucosa and submucosa, marked oedema of the submucosa and infiltration with eosinophils and to a lesser extent with mononuclear cells and polymorphonuclear cells, without evidence of vasculitis. These reactions were compatible with a type I hypersensitivity reaction in the intestinal wall.

3 A third pattern of histopathology was seen in three patients who had a preceding illness. Their clinical and histopathological findings were as follows:

Case 1 Necrotising ileitis in a 60 year old male with cardiovascular insufficiency for two weeks preceding the intestinal lesions; necrosis of the mucosa and submucosa, vascular congestion, marked oedema of the submucosa, no evidence of vasculitis or cell infiltration.

Case 2 Necrotising jejunitis in a 65 year old male after hypotension and shock with traumatic rupture of the spleen, fracture of the pelvis and shoulder resulting from a fall from a tree; haemorrhagic necrosis of the mucosa-submucosa, congestion, and haemorrhage marked at the tips of the villi, oedema of the submucosa and muscle layers, few polymorphonuclear leucocytes in the submucosa, no evidence of vasculitis. The serosal surface of the jejunum was unaffected.

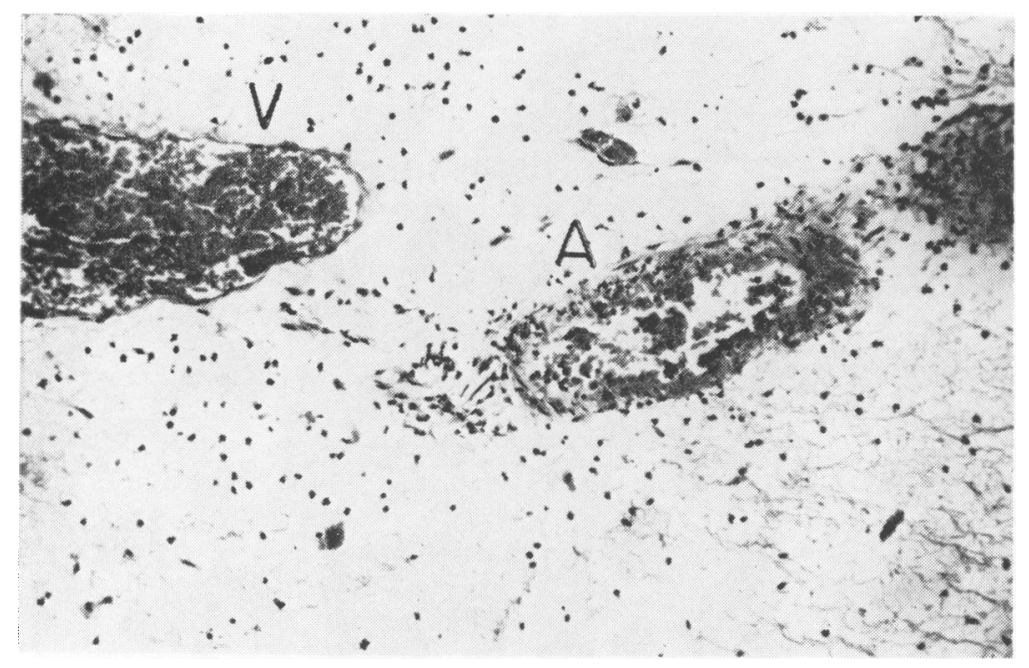

Fig. 8 Polymorphonuclear and mononuclear ce!l infiltration in wall of small artery $(A)$ in oedematous submucosa. Necrotising jejunitis and ileitis in a 64-year-old female with Ascaris lumbricoides in the intestine and anti-Ascaris antibody in the serum. Note venule is unaffected. $H$ and $E$. $\times 625$. 
Case 3 Necrotising ileitis in a 11 day old premature infant with abdominal distension, blood-stained discharge per anum; patchy gangrene in the proximal ileum; necrosis of the mucosa-submucosa, oedema of the submucosa, no evidence of vasculitis or cell infiltration.
The mesentery and its blood vessels in all three cases were macroscopically normal.

\section{Extraintestinal lesions}

Necropsy examination of the extraintestinal organs and tissues was possible in 17 cases. In seven out of

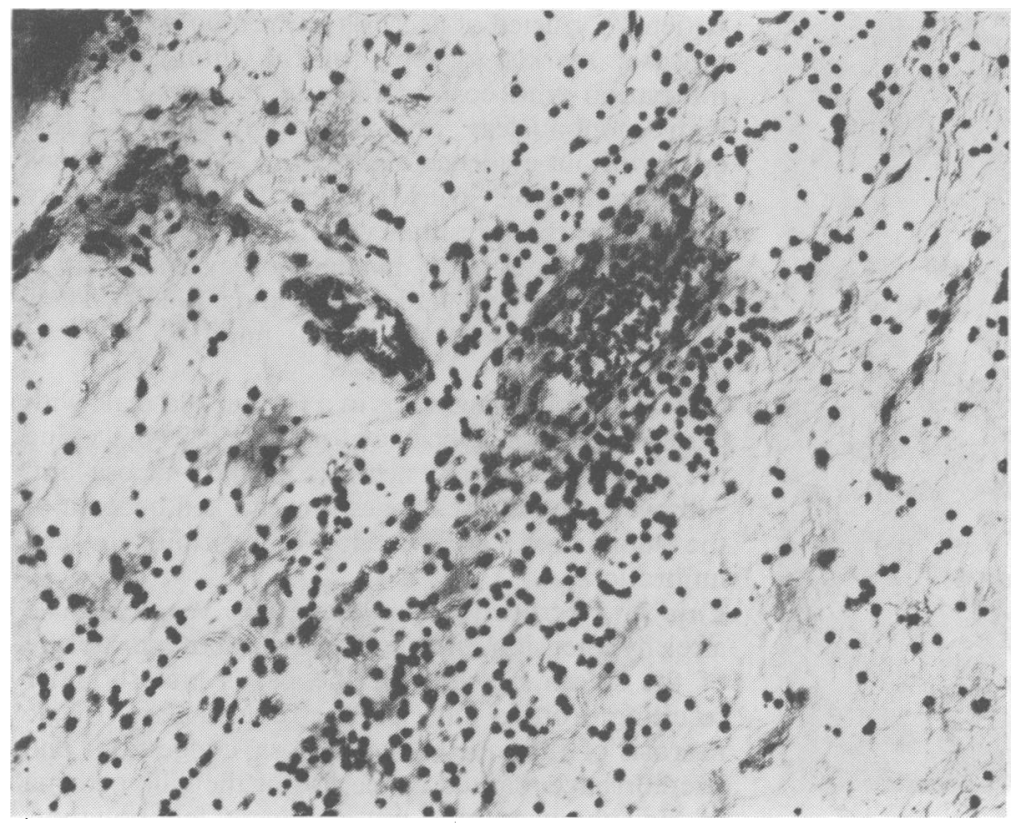

Fig. 9 Infiltrate of mononuclear cells around small artery in submucosa. Necrotising jejunitis and ileitis in a 5-year-old boy. $H$ and $E, \times 190$.

Fig. 10 Hyaline thrombi in small vein of submucosa. Necrotising jejunitis in a 38-yearold female. $H$ and $E, \times 625$.

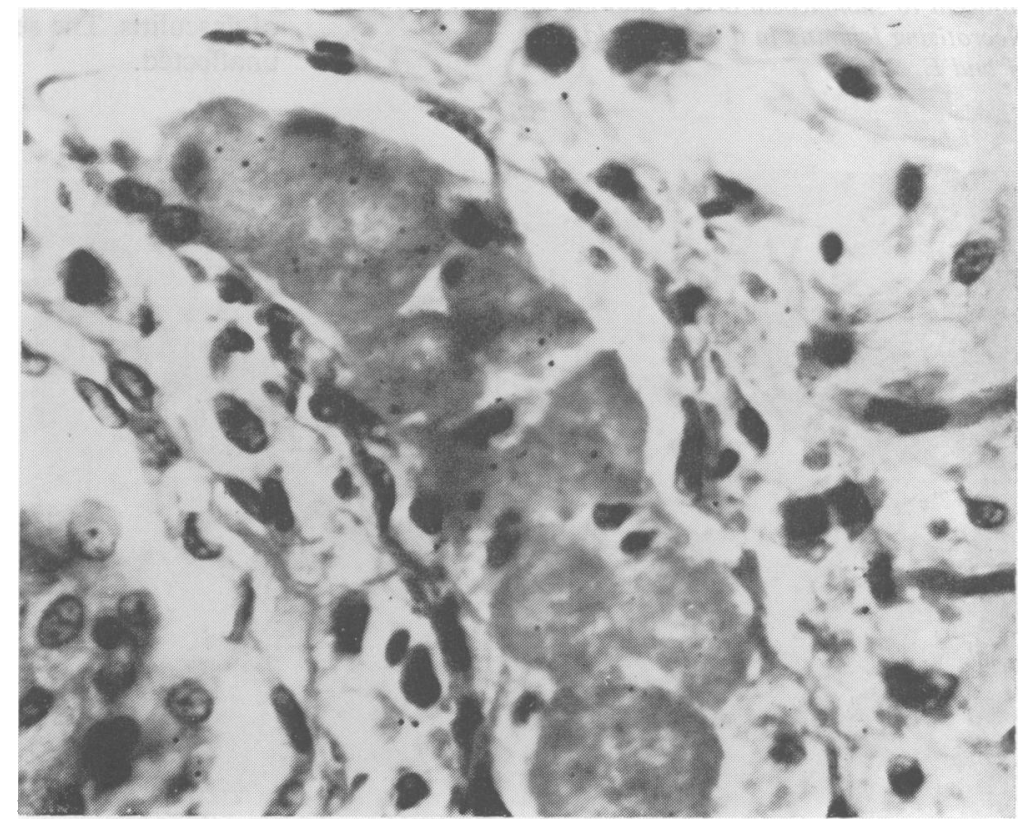




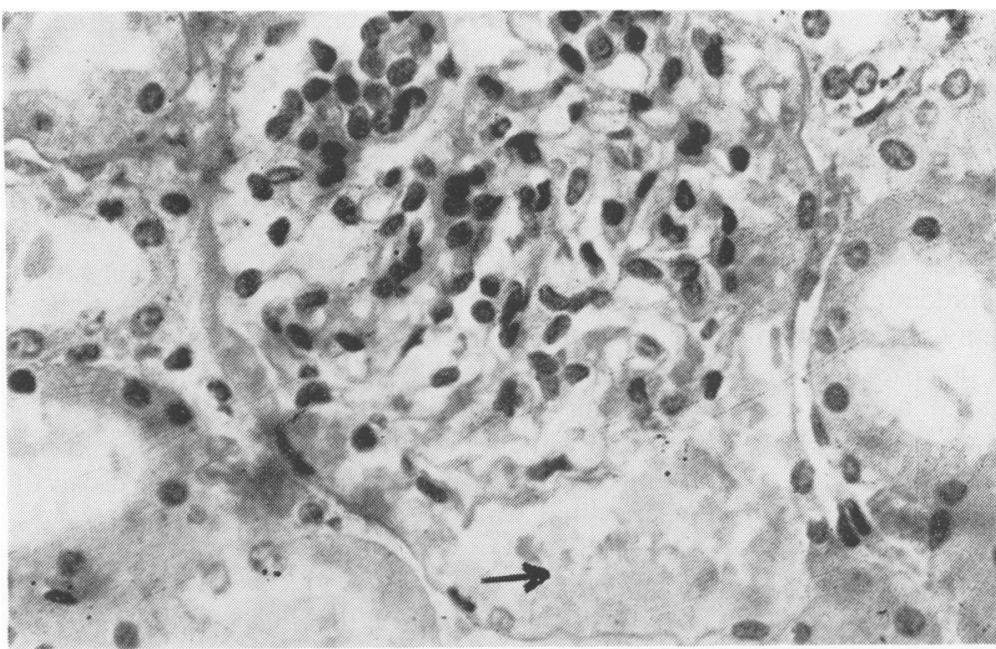

Fig. 11 Amorphous material (arrow) in Bowman's capsular space. Necrotising jejunitis. $H$ and $E, \times 625$.

11 patients in whom arteritis or fibrinoid necrosis of the small arteries and cell infiltration of the submucosa were present, the following extraintestinal abnormalities were noted: congestion of the glomeruli and intertubular regions of the kidney, hypercellularity of the glomeruli without polymorphonuclear cell infiltration, hyaline or granular casts in the renal tubules, the presence of granular or eosinophilic material in Bowman's capsular space (Fig. 11) and in two of these cases there was mononuclear cell infiltration of the portal tracts of the liver. In six other cases (three children and three adults), congestion and oedema of the lung were noted.

In two cases with intestinal arteritis, the small arteries of the corresponding mesentery also showed acute arteritis with polymorphonuclear cell infiltration of the vessel wall and perivascularly. Three adult patients with arteritis in the intestinal vessels showed oedema of the brain, while one 7 year old child with extensive necrotising jejunitis with arteritis is recorded as having localised fits of the upper limbs.

\section{Lymph node histology}

The histology of the mesenteric lymph nodes was studied in 26 cases. In 20 moderate to intense reactions indicative of a humoral immune response was noted, while in three of these a mild cellmediated immune response was also evident. Three cases showed equally marked humoral and cellmediated immune response, while in two cases it was predominantly cell mediated.

Of 15 cases with intestinal arteritis, 12 cases had moderate or marked humoral immune response, while in three cases with intestinal histopathology compatible with a type I hypersensitivity reaction, one had a humoral immune response.

Lymph node eosinophilia was present in two children who also had intense infiltration of the intestinal submucosa with eosinophils; one of these also had a heavy load of Ascaris lumbricoides and hookworms. In both these cases the intestinal histopathology resembled that of a type I hypersensitivity reaction.

\section{Cell counts in peripheral blood}

Counts were available in 34 cases. A polymorphonuclear leucocytosis was present in 32. Blood eosinophilia $(5180 / \mathrm{c} \mathrm{mm})$ was present in one case which also had intestinal roundworms and fibrinoid necrosis of intestinal arteries without intestinal eosinophilia. In seven cases with intestinal eosinophilia, none showed blood eosinophilia.

In 37 cases with intestinal arteritis, the patients ages ranged from $2 \frac{1}{2}$ to 64 years; the oldest patient was a 64 year old female with intestinal ascariasis, precipitating antibody against larval antigens of $A$. lumbricoides and was a vegetarian. The mortality in this group was $27 \%$.

In 12 of the patients with intestinal arteritis, $A$. lumbricoides was present in the intestinal lumen, while these worms were present in three out of eight patients with intestinal histopathology suggestive of a type I hypersensitivity reaction.

\section{Discussion}

Necrotising enteritis has been described in the literature as having occurred in neonates, especially in those born under conditions of stress, in adults with various associated conditions such as cardio- 
vascular insufficiency, severe infections, surgical operations, extensive trauma and shock, and in apparently healthy adults and children from diverse geographical and environmental situations. There is a wide variation in the factors-diet, season, associated illnesses, site of intestinal involvementwhich are associated with necrotising enteritis and these seem to depend on whether it is sporadic and more based on host factors 'or epidemic. Correspondingly, there have been many theories to account for the pathogenesis of necrotising enteritis; there are two broad categories of aetiological factors.

DISTURBANCES OF THE INTESTINAL

MICROCIRCULATION

Functional circulatory states

Such factors as reflex diversion of blood from the intestine resulting in hypoperfusion and ischaemic necrosis are particularly relevant to necrotising enteritis in stressed neonates. ${ }^{9}$ In both humans ${ }^{10}$ and in experimental animals ${ }^{11}$ shock has been shown to result in haemorrhagic necrosis in the inner intestinal wall in which the brunt of the damage was suffered by the mucosa-submucosa. Sandritter ${ }^{11}$ noted the predominance of the lesions in the duodenum and jejunum (see also Robinson and Mirkovitch. ${ }^{12}$ )

The occurrence of jejunal lesions of functional circulatory origin after extensive traumatic injury and shock is illustrated by case 2 described above; these lesions resemble the experimental lesions reported by Sandritter. ${ }^{11}$ Cases 1 and 3 probably illustrate the operation of functional circulatory insufficiency in precipitating intestinal ischaemia and necrosis. The similarity of the histopathology in these three cases parallels the striking similarity between many aspects of neonatal necrotising interitis on the one hand and of necrotising enteritis in aged patients with heart failure and low flow states on the other. ${ }^{13}$

A significant finding in some of our mild and early cases was the focal distribution of the mucosalsubmucosal necrosis, without evidence of thrombosis suggesting the operation of functional microcirculatory disturbances.

\section{Obstructive vascular factors}

Obstruction of the larger intestinal blood vessels has been recorded as leading to necrotising enteritis. Organic obstruction of the microcirculation by thrombi after disseminated intravascular coagulation has been described in necrotising enteritis; however, it is likely that such thrombosis is secondary to intestinal necrosis and the liberation of tissue thromboplastic substances, endotoxin, Gram negative bacteraemia or 'low flow states' and not the primary cause of the intestinal lesions.

\section{Infective factors}

It is significant that neonatal necrotising enteritis occurs at a time when the gut is first colonised by bacteria. ${ }^{14}$ Shwartzman reactions, ${ }^{15}$ endotoxin mediated vasoconstriction, ${ }^{16}$ or a direct necrotising action of intestinal bacteria ${ }^{17} 18$ have been proposed as aetiological factors. Bacterial pathogens have been isolated from the blood and peritoneal fluid in necrotising enteritis ${ }^{19}$ although the presence of bacteria or their products in the circulation could probably be due to the devitalisation and necrosis of the intestinal wall. ${ }^{19}$

\section{Cl. welchii}

There are two aspects of the role of $\mathrm{Cl}$. welchii in necrotising enteritis (1) as an initiator of the intestinal necrosis and (2) in the colonisation of already established necrotic foci, to produce intestinal gas gangrene. Murrell et al. ${ }^{4}$ suggested a direct necrotising action of $\mathrm{Cl}$. welchii. Cultures of this organism have produced necrotising enteritis in experimental animals. ${ }^{20} 21$

Cl. welchii type C (including former 'type F') has been incriminated in 'darmbrand' and 'pig-bel' enteritis in previously healthy persons; the necrotising $\beta$ toxin of this organism is of sufficient potency to initiate the intestinal necrosis. $\mathrm{Cl}$. welchii type $\mathrm{A}$, on the other hand, which lacks the $\beta$ toxin, may need synergistic factors such as $\mathrm{pH}$ alteration, stasis-for example, Hirschsprung's disease or ileus-alterations in the bacterial flora, and an already established focus of necrosis for colonisation and the production of intestinal gas gangrene which is the essential lesion in this disorder.

None of our cases was associated with the consumption of sweet potato, which Lawrence and Walker ${ }^{8}$ regarded as acting synergistically with clostridial toxins, through its trypsin inhibitors.

Either as an initiator of the intestinal lesion or as a secondary invader of an already existing necrotic focus there is impressive evidence in the literature for the role of $\mathrm{Cl}$. welchii in necrotising enteritis. This consists of (1) epidemiological data from the New Guinea epidemics of 'pig-bel' and the isolation of a single serotype of $\mathrm{Cl}$. welchii from the epidemic and sporadic cases and from the pork or other food which precipitated the illness; (2) significantly raised and rising titres of clostridial antitoxins; (3) the efficacy of passively administered clostridial antitoxins in established cases of necrotising enteritis from which clostridia have been recovered; and (4) the experimental reproduction of the disorders in animals by the feeding of cultures of $\mathrm{Cl}$. welchii. 
Ancillary evidence is the presence of large Gram positive bacilli, morphologically resembling clostridia, in the intestinal wall in necrotising enteritis and pneumatosis intestinalis which is probably due to intramural gas production by invading bacilli. ${ }^{32}$ Our observation of the presence of bacilli which morphologically resembled clostridia in the contents and in the wall of the diseased jejunum and upper ileum in which these bacilli are normally absent, and the significantly higher antitoxin titres in NE patients, supports a pathogenic role of $\mathrm{Cl}$. welchii in the second stage (see below) of necrotising enteritis. Histologically intense oedema and necrosis in the intestinal wall would be compatible with the action of clostridial toxins.

Koslowski and co-workers ${ }^{23}$ demonstrated that strains of $\mathrm{Cl}$. welchii from necrotising enteritis had a greater capacity for producing histamine than strains from other sources. Our results confirm this finding. Masses of bacilli which we observed adhering to the mucosal lining and crypts and in the wall of the intestine, could aggravate the microcirculatory disturbances in the adjacent wall by excessive histamine production. The $\mathrm{Cl}$. welchii 'enterotoxin' which is responsible for fluid accumulation in experimental ileal loops, is of doubtful relation to necrotising enteritis, as, apart from an accumulation of fluid, no gross signs of haemorrhagic inflammation as seen in necrotising enteritis are produced by the enterotoxin. ${ }^{23 a}$ Conversely, fluid accumulation in the intestine and watery diarrhoea are not features of necrotising enteritis.

Other factors which are reported to be possible synergistic factors are reduced intestinal mucus secretion, absence of phagocytic and antibody immunity, hyperosmolar feeds in infants, and voluminous carbohydrate meals.

\section{HYPERSENSITIVITY REACTIONS}

The normal intestinal wall has been documented as a tissue of high immunological reactivity. It shares with the respiratory tract the possession of relatively high numbers of IgE producing cells ${ }^{24}$ and there is increasing evidence for the participation of the gastrointestinal tract in allergic reactions. ${ }^{25}$ Hypersensitivity induced in animals to parasitic antigens may provide useful models in the aetiology of some of our cases. Litt ${ }^{26}$ considered that parasitic infestation was one of the prime examples of eosinophilic inflammation.

\section{Type I hypersensitivity}

Nematode antigens are particularly effective inducers of an IgE response. Johansson et al. ${ }^{27}$ found that Ethiopian children with ascariasis had IgE levels which were 28 times higher than in control Swedish children. Many authors have described type I reactions to nematode sensitised experimental animals; in the gut these reactions were most marked at the tips of the villi leading to shedding of the epithelium.

Apart from being a possible source of antigen in necrotising enteritis, ascarid worms may also liberate vasoactive amines non-immunologically by the direct degranulation of intestinal mast cells. ${ }^{28}$ The majority of our cases of necrotising enteritis had lesions localised in the small intestine and it is relevant to a possible aetiological role of ascarids that these worms are also localised in this part of the intestine.

The lesions in those of our cases which were suggestive of a type I reaction were oedema and infiltration of eosinophils and mononuclear cells into the submucosa in the vicinity of the necrotic tissue. Infiltration of eosinophils occurs into areas which are the site of an antigen-antibody reaction, ${ }^{29}$ where IgE is the most likely class of antibody involved. ${ }^{30}$ In some of our cases the lesions consisted of oedema and haemorrhage in the submucosa with hyaline thrombosis in the smaller submucous vessels in the absence of vasculitis and are similar to those described in protracted anaphylaxis. ${ }^{30}$

Antigens from other intraluminal sources such as bacteria or food could also mediate these reactions. Thomlinson and Buxton ${ }^{31}$ suggested that haemorrhagic enteritis in calves and oedema disease of the intestine in swine, the histology of which bears a close resemblance to those in necrotising enteritisoedema, haemorrhage, thrombosis, mononuclear cell infiltration, and local eosinophilia-are caused by anaphylactic hypersensitivity to $E$. coli antigens.

\section{Type III hypersensitivity reactions}

Arthus-like reactions in the intestine in cases of necrotising enteritis were noted by Welch and Sumitswan ${ }^{32}$ in two of their cases, although their significance was not commented on. These authors found parasites including nematodes in the stools of 10 out of 27 of their patients; this incidence was not higher than that found in the general population and they concluded that there was no evidence of an aetiological role of parasites in this disease. As in our series, all their cases were confined to the small intestine.

Trichinosis in man produces eosinophilia and arteritis similar to that seen in serum sickness ${ }^{33}$ and it is tempting to speculate that the link between such lesions in trichinosis and the vasculitis in Northern Thailand, as reported by Welch and Sumitswan, ${ }^{32}$ is the frequency of the consumption of undercooked pork in this region. 
Ishikura et al. $^{34}$ described intestinal anisakiasis which produces an illness like acute regional ileitis with intestinal lesions resembling Arthus reactions, induced by the nematode larvae.

Although classical descriptions of vasculitis of immune complex disease include polymorphonuclear cell infiltration of the perivascular regions, the age of the lesions and the composition of the antigenantibody complex, among other unidentified factors, may modify this reaction, resulting in a paucity of polymorphonuclear cells, ${ }^{35}$ a predominantly mononuclear cell infiltration, ${ }^{36}$ or the presence of fibrinoid necrosis with little or no cellular infiltration in or around the vessels. ${ }^{37}{ }^{38}$ These three types of reactions were seen in our cases and are compatible with type III reactions of varying duration.

The vasculitis in our cases appeared to be confined to the small arteries and would probably represent the serum sickness type of arteritis rather than the classical local Arthus reaction, in which the venules and capillaries are predominantly affected ${ }^{39}$ or cellular hypersensitivity in which the lesions are mainly perivenous. ${ }^{30}$

The idea that the intestinal lesions in some of our cases were a component of a disease resembling serum sickness is supported by the finding of extraintestinal lesions which are similar to those described in classical serum sickness. ${ }^{30}$ These include the presence of amorphous material in Bowman's capsular spaces, hyaline and red cell casts in the renal tubules, hypercellularity of the glomeruli, and mononuclear cell infiltration of the portal tracts of the liver. In one adult patient with intestinal vasculitis and extensive gangrene of the jejunum and upper ileum, the clinical symptoms of peripheral neuritis were seen; neuritis of the Guillain Barré type and brachial neuritis have been described as frequent complications of primary serum sickness. In addition, this patient showed vascular congestion of the glomeruli and intertubular regions of the kidney, with eosinophilic material in the renal tubules suggestive of casts, hypercellularity of the glomeruli and mononuclear cell infiltration of the hepatic portal tracts. In a second case, jaundice was present in a 30 year old female with necrosis and oedema of the mucosa-submucosa and thrombosis of the submucous vessels, who had similar extraintestinal lesions. A third case, a 7 year old child with extensive gangrene of the jejunum and ileum, round worms in the intestinal lumen, and vasculitis of the intestinal arteries, showed similar extraintestinal histopathology. On the other hand, in two patients who had intestinal histopathology which was suggestive of a type I instead of a type III reaction, no systemic lesions of the immune complex type were seen.
INTESTINAL LOCALISATION OF

HYPERSENSITIVITY REACTIONS

The greater sensitivity of the jejunal mucosa to ischaemia may depend on microvascular factors which may also be the basis for the predominance of vasculitis in the jejunum. The fact that vasculitis was most marked in the small intestine could perhaps also be explained on the basis of the findings of Cochrane and Henson ${ }^{40}$ that a concomitant local type I hypersensitivity reaction with the resultant increase in vascular permeability may make it easier for circulating immune complexes to penetrate the endothelium. The participation of a type I reaction in our case of necrotising enteritis has already been suggested and we would add that the non-immunological release of histamine by intestinal nematodes, or even by the histidine decarboxylase of intestinal bacteria, may also contribute to the increased permeability of the intestinal microcirculation.

\section{Shwartzman reactions}

The vascular histopathology in our cases would appear to resemble immune complex reactions rather than Shwartzman reactions because of the presence of oedema and eosinophil infiltration which was sometimes very marked, the localisation of the reactions to small arteries rather than to small veins and capillaries, and the well-marked infiltration of the arterial walls by polymorphonuclear and mononuclear cells without coextensive thrombosis.

In conclusion we would regard necrotising enteritis as a two-stage process: in stage 1 , a necrotic focus is established in the intestinal wall by ischaemia due to functional or organic causes or to necrotising factors of bacterial origin. Stage 2 is the final common path of necrotising enteritis, irrespective of the mechanism that was responsible for the initiation of the necrosis. The extension of the necrosis to gangrene, toxaemia, and death of the patient will depend upon the toxigenic capacity of the invading bacterium. It is the second stage which is apparently referred to as necrotising enteritis, irrespective of the nature of the agents which precipitated it and it is essentially gas gangrene of the intestinal wall. This differentiation recognises 'initiating' factors in the intestinal necrosis and 'promoting' factors which contribute to the establishment of the necrosis in stage 1 or the evolution of stage 1 to stage 2 .

In addition to the many aetiological factors which have been proposed in the literature as initiating stage 1 , we would suggest from our findings that hypersensitivity reactions of type I and III are also responsible for obstructive vascular effects and that the source of the antigens is probably the intestinal lumen. The antigen challenge to an already sensitised intestine could possibly arise from extraintestinal 
sources such as from migrating Ascaris larvae in the respiratory tract ${ }^{25}$ or from the intestinal lumen.

Intestinal vasculitis in necrotising enteritis has apparently been reported only from Sri Lanka and Thailand. Intestinal parasites which are common in these two countries may be the source of the antigens which cause these reactions.

The various aetiological factors would vary in importance with each sporadic case and their operation would depend on many factors such as the age of the patient, the presence of intestinal parasites, systemic circulatory disorders, and the nature of the diet.

The authors gratefully acknowledge the assistance of Dr K M Pavri (Virus Research Centre, Poone, India), Professor C Barr Kumarakulasinghe, Dr C Ratnatunga, and Dr M N Prematilleke of the Kandy General Hospital. The study was supported by grants from the National Science Council, Sri Lanka, and the University of Sri Lanka, Peradeniya.

\section{References}

${ }^{1}$ Ratnatunga PCA, Panabokke RG, Kumarakulasinghe CB, Eaton HL, Tharmarajah A. Necrotising enteritis in Ceylon. Proc Ceylon Assoc Adv Sci 1969; 25: 7-8.

${ }^{2}$ Wijesundera P de S. Inflammation of the small bowel. J Colombo General Hosp 1972; 3: 78-84.

${ }^{3}$ Kumarakulasinghe $\mathrm{CB}$, Ratnatunga C. A study of necrotising enteritis. Proc Sri Lanka Assoc Adv Sci 1977; 32: 13.

${ }^{4}$ Murrell TGC, Roth L, Egerton J, Samels J, Walker PD. Pig bel: enteritis necroticans: a study in diagnosis and management. Lancet 1966; 1: 217-22.

${ }^{5}$ Cottier H, Turk J, Sobin L. A proposal for a standardized system of reporting human lymph node morphology in relation to immunological function. J Clin Pathol 1977; 26: 317-31.

${ }^{6}$ Cowan ST, Steel KJ. Manual for the identification of medical bacteria. Cambridge: Cambridge University Press, 1965.

'Eggleston LV. Effect of cations on amino acid decarboxylases. Biochem J 1958; 68: 557-60.

${ }^{8}$ Lawrence G, Walker PD. Pathogenesis of enteritis necroticans in Papua New Guinea. Lancet 1976; 1: 125-6.

${ }^{9}$ Lloyd JR. The etiology of gastrointestinal perforations in the new born. $J$ Pediatr Surg 1969; 4: 77-84.

${ }^{10}$ Sandritter W, Lasch HG. Pathological aspects of shock. Meth Achievm Exp Path 1967; 3: 86-121.

${ }^{11}$ Sandritter W. Shock induced lesions in the small intestines in experimental animals. In: Thackray AC Avery Jones F, eds. The small intestine. Oxford: Black wells, 1965: 87-94.

${ }^{12}$ Robinson JWL, Mirkovitch V. The recovery of function and microcirculation in small intestinal loops following ischaemia. Gut 1972; 13: 784-0.

${ }^{13}$ Silen W. Editorial comment. In: Schwartz SI et al., eds.
Year book of surgery. Chicago: Year Book Medical Publishers, 1973: 350.

${ }^{14}$ British Medical Journal. Neonatal necrotizing enterocolitis (leading article). Br Med $J$ 1970; 3: 121-2.

${ }^{15}$ Hermann RE. Perforation of the colon from necrotizing colitis in the new born: report of a survival and a new aetiologic concept. Surgery 1965; 58: 436-41.

${ }^{16}$ Mizrahi A, Barlow O, Berdon W, Blanc WA, Silverman WA. Necrotizing enterocolitis in premature infants. J Pediatr 1965; 66: 697-706.

${ }^{17}$ Killingback MJ, Lloyd Williams K. Necrotizing colitis. Br J Surg 1961; 49: 175-85.

${ }^{18}$ Stein H., Beck J., Solomon A, Schmaman A. Gastroenteritis with necrotizing enterocolitis in premature babies. Br Med J 1972; 2: 616-9.

${ }^{19}$ British Medical Journal. Necrotizing enterocolitis in premature infants (leading article). Br Med J 1966; 2: 1089-90.

${ }^{20}$ Field HI, Goodwin RFW. The experimental reproduction of enterotoxaemia in piglets. J Hyg (Camb) 1959; 57: 81-91.

${ }^{21}$ Egerton JR. Bacteriology of enteritis necroticans in New Guinea highlanders. Papua New Guinea Med J 1966 9: 55-9.

${ }^{22}$ Pedersen PV, Hansen FH, Halveg AB, Christian ED, Justeson T, Hogh P. Necrotising enterocolitis of the new born. Is it gas gangrene of the bowel? Lancet 1976; 2: 715-6.

${ }^{23}$ Koslowski L, Schneider H, Heise C. Experimentelle untersuchungen uber die Histaminbildung des Bac. enterotoxicus im Vergleich mit anderen pathogenen Anaerobiern. Klin Wochenschr 1951; 29: 29-30.

23aHauschild AHW, Niilo L, Dorward WJ. Clostridium perfringens type $\mathrm{A}$ infection of ligated intestinal loops in lambs. Appl Microbiol 1968; 16: 1235-39.

${ }^{24}$ Ishizaka H. Human reaginic antibodies. Ann Rev Med 1970; 21 : 187-200.

${ }^{25}$ Lancet Allergy in the gastrointestinal tract (editorial). Lancet 1975; 2: 1021-3.

${ }^{26}$ Litt $M$. Eosinophils and antigen antibody reactions. Ann N Y Acad Sci 1964; 116: 964-85.

${ }^{27} \mathrm{~J}$ ohansson SGO, Mellbin T, Vahlquist Bo. Immunoglobin levels in Ethiopian preschool children with special reference to high concentrations of immunglobulin E (IGND). Lancet 1968; 1: 1118-21.

${ }^{28}$ Uvnas B, Diamant B, Hogberg B, Thon IL. Mechanism of mast-cell disruption induced by a principle extracted from Ascaris suis. Am J Physiol 1960; 199: 575-8.

${ }^{29} \mathrm{Litt}$ M. Studies in experimental eosinophilia IX. Inhibition by puromycin of the eosinophil response which hemocyanin elicits in guinea pig lymph nodes J Immunol 1972; 109: 222-6.

${ }^{30}$ Waksman BH. An atlas of experimental immunology and immunopathology. New Haven: Yale University Press, 1970.

${ }^{31}$ Thomlinson JR, Buxton A. A comparison of experimental anaphylactic shock in guinea pigs with naturally occuring oedema disease and haemorrhagic gastroenteritis in pigs. Res Vet Sci 1962; 3: 186-202.

${ }^{32}$ Welch TP, Simitswan S. Acute segmental ischaemic enteritis in Thailand. Br J Surg 1975; 62: 716-9. 
${ }^{33}$ Rose B. Editorial comment. In: Samter $M$ et al., eds. Immunological diseases. Boston: Little, Brown, 1965: 470.

${ }^{34}$ Ishikura H, Hayasaka H, Kikuchi Y. Acute regional ileitis at Iwanai in Hokkaido with special reference to intestinal anisakiasis. Sapporo Med J 1967; 32: 183-96.

${ }^{35}$ Parish WE, Rhodes EL. Bacterial antigens and aggregated gamma globulin in the lesions of nodular vasculitis Br J Dermatol 1967; 79: 131-47.

${ }^{36}$ Gell PGH, Hinde IT. Observations on the histology of the Arthus reaction and its relation to other known types of skin hypersensitivity. Int Arch Allergy Appl Immunol 1954; 5: 23-46.

${ }^{37}$ Cochrane CG, Weigle WO. The cutaneous reaction to soluble antigen-antibody complexes. A comparison with the Arthus phenomenon. J Exp Med 1958: 108: 591-604.

${ }^{38}$ Germuth FG Jr, Pollack AD. The production of lesions of 'serum sickness' in normal animals by the passive transfer of antibody in the presence of antigen. Bull Johns Hopkins Hosp 1958; 102: 245-62.

${ }^{39}$ Cochrane CG. The Arthus reaction. In: Zweifach BW, Grant L, McCluskey RT, eds. The inflammatory process. New York: Academic Press, 1965: 615.

${ }^{40}$ Cochrane CG, Henson PM. Experimental immune complex disease. In: Turk JL, Bonomo L, eds. Immune complex diseases. Milan: Carlo Erba Foundation, 1970: 11. 